\title{
Peak to Average Power Ratio Performance of a 16-QAM/OFDM System with Partial Transmit Sequence
}

\author{
Amritbir Kaur $^{1}$, Er. Harshit ${ }^{2}$ \\ ${ }^{I}$ (Mtech Student, ECE Department, DIET, Kharar, Mohali) \\ ${ }^{2}$ (HOD and AP, ECE Department, DIET, Kharar, Mohali)
}

\begin{abstract}
Orthogonal Frequency Division Multiplexing is a spectral efficient transmission format. But it suffers from the problem of high Peak to Average Power Ratio. This high Peak to Average Power Ratio leads the power amplifier into saturation and results in non-linear distortion at the output of power amplifier. Different peak to average power ratio reduction techniques are available in literature. This paper computes the performance of partial transmit scheme, which is one of the important peak to average power ratio reduction technique
\end{abstract}

Keywords: Orthogonal Frequency Division Multiplexing, Partial Transmit Sequence, Peak to Average Power Ratio, Power Amplifier, Scrambling Techniques.

\section{INTRODUCTION}

R.W. Chang proposed the first Orthogonal Frequency Division Multiplexing (OFDM) technique in 1966 for fading limited channels. Since then it has emerged as a very attractive and powerful transmission technique. It provides transmission of multiple signals simultaneously, over a single transmission path. In OFDM a high rate data stream is divided into many low data streams these steams are then multiplied by corresponding carrier frequency signals that are orthogonal to each other. A composite signal so formed by multiplexing these modulated signals is called the OFDM signal. For the understanding purpose, OFDM can be thought of as a special case of Frequency Division Multiplexing (FDM) with the subcarriers which are orthogonal to each other. The lower rate data stream so formed from the higher rate data stream (after serial to parallel conversion) has large symbol duration so Inter Symbol Interference (ISI) is reduced in OFDM. In other words, there is less dispersion in time domain due to multipath delay spread as in Time Division Multiplexing (TDM) [1].

The condition for maintaining the orthogonality is that the frequency spacing between the carrier signals must be an integer multiple of the lowest carrier frequency. That is, each subcarrier has an integer number of cycles in time period $\mathrm{T}$. The numbers of cycles in adjacent subcarriers differ by exactly one. This technique provides high data rate even if relatively small frequency bandwidth is available. Also, OFDM based system has other favorable properties such as high spectral efficiency, robustness to channel fading and impulse interference. Because of these advantages, OFDM has been adopted in both wireless and wired applications in recent years. OFDM-based systems are more immune to impulse noise, fast fades and can be of greater pursuit for wireless applications as they eliminate the need for equalizers. Also, efficient hardware implementations can be realized using Fast Fourier Transform (FFT) techniques for small numbers of carriers. So, OFDM has emerged as the standard of choice in a number of important high data applications in past few decades.

High Peak to Average Power Ratio is one of the major practical complications of the uncoded OFDM signals. PAPR is defined as the ratio between the instantaneous power of the peaks and the average power of the signal. Mathematically,

$$
\text { PAPR }=\frac{\max [\mathrm{x}(\mathrm{t})]^{2}}{\mathrm{E}\left\{|\mathrm{x}(\mathrm{t})|^{2}\right\}}
$$

Where $\mathrm{E}\{$.$\} is the expectation operator. Also,$

Crest Factor $=\sqrt{P A P R}$

PAPR is usually analyzed by using statistical parameter called complementary cumulative distribution function (CCDF). CCDF shows the probability that PAPR is above a threshold level. CCDF is defined as

$P(P A P R \geq z)=1-\left((1-\exp (-\mathrm{z}))^{\mathrm{N}}\right.$

When the $\mathrm{N}$ sinusoidal signals after modulation by their carriers, adds mostly constructively the peak envelop power is as much as $\mathrm{N}$ times the mean power. As a result the amplitude of such a signal can have very large values. When high-peak power signals pass through power amplifiers, Analog to Digital (A/D) and Digital to Analog (D/A) converters, peaks are distorted non-linearly because of amplifier and converters imperfection thus, the output signal will suffer from intermodulation distortion resulting in energy being generated at frequencies outside the allocated bandwidth. Therefore average signal power must be kept low in order to 
prevent the transmitter amplifier and other circuitry limiting. Minimizing the PAPR allows a higher average power to be transmitted for a fixed peak power, improving the overall signal to noise ratio at the receiver [2]. PAPR reduction methods can be categorized as distortion type and non-distortion type methods. Distortion type techniques reduce the PAPR by reducing the higher peaks in the signal and thereby distorting the signal. In other words, these methods modify the signals in time domain to reduce the PAPR so that a certain level of distortion is introduced. These methods cause an increase in both In-Band Radiation (IBR) and Out of Band Radiation (OBR) and Bit Error Rate (BER). Example: clipping, peak windowing, peak cancellation etc.Nondistortion type techniques include coding and data scrambling methods. These methods do not introduce any distortion, but they also do not ensure reduced PAPR. So they must be used along with other distortion type techniques. They do not cause high OBR and BER. Example: peak reduction carrier, selective level mapping (SLM), partial transmit sequence (PTS) and block coding [3].

\section{Partial Transmit Sequence}

The partial transmit sequence (PTS) technique partitions an input data block of $\mathrm{N}$ symbols into $\mathrm{V}$ disjoint subblocks as follows:

$$
X=\left[X^{0}, X^{1}, X^{2}, \ldots, \mathrm{X}^{v-1}\right]^{T}
$$

where $X^{i}$ are the subblocks that are consecutively located and also are of equal size. Unlike the SLM technique in which scrambling is applied to all subcarriers, scrambling (rotating its phase independently) is applied to each subblock [5] in the PTS technique. Then each partitioned subblock is multiplied by a corresponding complex phase factor $b^{v}=e^{j \phi v}, v=1,2, \ldots, V$, subsequently taking its IFFT to yield

$$
X=I F F T\left\{\sum_{v-1}^{V} b^{v} X^{v}\right\}=\sum_{v-1}^{V} b^{v} . I F F T\left\{X^{v}\right\}=\sum_{v-1}^{V} b^{v} X^{v}
$$

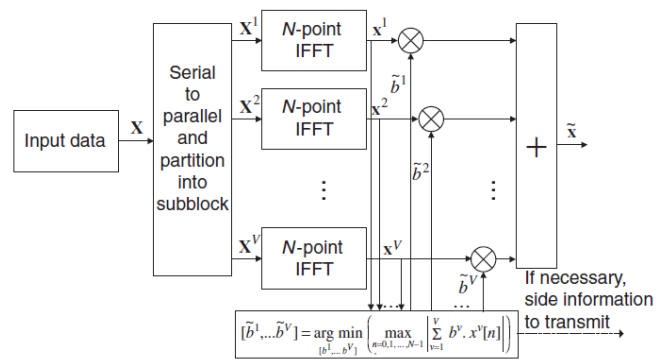

Figure 1 Block diagram of partial transmits sequence (PTS) technique for PAPR reduction.

where $\left\{X^{v}\right\}$ is referred to as a partial transmit sequence (PTS). The phase vector is chosen so that the PAPR can be minimized [6], which is shown as

$$
\left[\tilde{b}^{1}, \ldots, \tilde{b}^{V}\right]={ }_{b^{1}, \ldots, b^{V}}^{\arg \min }\left(\max _{n=0,1, \ldots, N-1}\left|\sum_{v=1}^{V} b^{v} x^{v}[n]\right|\right)
$$

Then, the corresponding time-domain signal with the lowest PAPR vector can be expressed as

$$
\tilde{X}=\sum_{v=1}^{V} \tilde{b}^{v} X^{v}
$$

In general, the selection of the phase factors $\left\{b^{v}\right\}_{v=1}^{V}$ is limited to a set of elements to reduce the search complexity [4]. As the set of allowed phase factors is $b=\left\{e^{j 2 \pi i / w} \mid i=0,1, \ldots, W-1\right\}, w^{V-1}$ sets of phase factors should be searched to find the optimum set of phase vectors. Therefore, the search complexity increases exponentially with the number of sub blocks.

The PTS technique requires V IFFT operations for each data block and $\left[\log _{2} W^{v}\right]$ bits of side information. The PAPR performance of the PTS technique is affected by not only the number of sub blocks, V, 
and the number of the allowed phase factors, W, but also the subblock partitioning. In fact, there are three different kinds of the subblock partitioning schemes: adjacent, interleaved, and pseudo-random. Among these, the pseudo-random one has been known to provide the best performance [7].

\section{PTS Algorithm}

To As discussed in section II, the PTS technique suffers from the complexity of searching for the optimum set of phase vector, especially when the number of subblock increases. In the literature $[8,9]$, various schemes have been proposed to reduce this complexity. One particular example is a suboptimal combination algorithm, which uses the binary phase factors of $\{1,-1\}$ [8]. It is summarized as follows:

1. Partition the input data block into $\mathrm{V}$ subblocks as in Equation (4).

2. Set all the phase factors $\mathrm{b}^{\mathrm{v}}=1$ for $\mathrm{v}=1: \mathrm{V}$, find PAPR of Equation (5), and set it as PAPR min.

3. Set $\mathrm{v}=2$.

4. Find PAPR of Equation (3.27) with $\mathrm{b}^{\mathrm{v}}=-1$.

5. If PAPR $>$ PAPR_min, switch bv back to 1 . Otherwise, update PAPR min=PAPR.

6. If $\mathrm{v}<\mathrm{V}$, increment $\mathrm{v}$ by one and go back to Stepu.Otherwise, exit this process with the set of optimal phase factors $\tilde{b}$.

\section{Simulation Results}

An The number of computations for Equation (5) in this suboptimal combination algorithm is V, which is much fewer than that required by the original PTS technique (i.e., $\mathrm{V}<<\mathrm{W}^{\mathrm{V}}$.

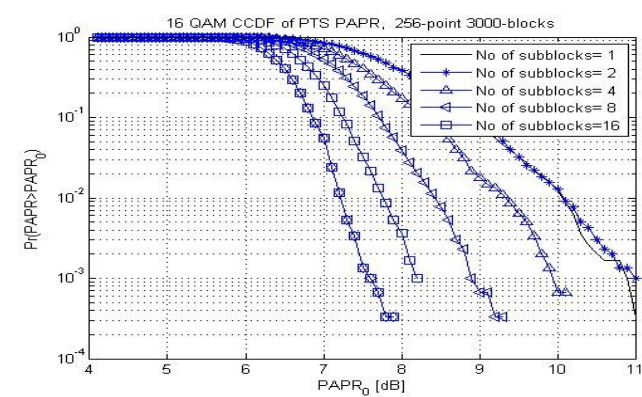

Figure 3 PAPR performance of a 16-QAM/OFDM system with PTS technique when the number of subblocks varies.

Figure 3 shows the CCDF of PAPR for a 16-QAM/OFDM system using PTS technique as the number of subblock varies. It is seen that the PAPR performance improves as the number of subblocks increases with $\mathrm{V}=$ $1,2,4,8$, and 16 .

\section{Conclusion}

Signal scrambling techniques work with side information which minimized the effective throughput since they commence redundancy. The signal scrambling techniques can be classified as Block coding, Partial Transmit Sequences (PTS), Tone Reservation (TR) and Tone Injection (TI), Selective Mapping (SLM), Interleaving and Active Constellation Extension (ACE). In this paper PTS technique has been implemented for different number of subblocks. From simulation results it has been concluded that as the number of subblocks increases, the value of PAPR decreases

\section{REFERENCES}

[1] Gordan A. Gow, Richard K. Smith (2006), Mobile and Wireless communications: An Introduction, New York: Mc Graw-Hill International.

[2] P. Muthu Chidambara Nathan (2008), Wireless Communications, New Delhi: PHI Learning Private Limited.

[3] N.Revueto (2008) PAPR reduction in OFDM systems. Master Thesis, Polytechnic University of Catalonia, Spain.

[4] Han, S.H. and Lee, J.H. (2005) An overview of peak-to-average power ratio reduction techniques for multicarrier transmission. IEEE Wireless Commun., 12(2), 56-65.

[5] Muller, S.H. et al. (1997) OFDM with reduced peak-to-average power ratio by multiple signal representation. In Annals of Telecommun., 52(1-2), 58-67.

[6] Muller, S.H. and Huber, J.B. (1996)OFDMwith reduced peak-to-average power ratio by optimum combination of partial transmit sequences. Electron. Lett., 32(22), 2056-2057.

[7] Muller, S.H. and Huber, J.B. (Sep. 1997)Anovel peak power reduction scheme for OFDM. PIMRC'97, vol. 3, pp. 1090-1094.

[8] Cimini, L.J. Jr (2000) Peak-to-average power ratio reduction of an OFDM signal using partial transmit sequences. IEEE Commun. Letters, 4(3), 86-88.

[9] Tellambura, C. (1998) A coding technique for reducing peak-to-average power ratio in OFDM. IEEE GLOBECOM'98, vol. 5, pp. $2783-2787$. 